Kontrazeption, Kinderwunsch und Schwangerschaftsplanung gehören in jede Erstuntersuchung psychisch kranker Frauen und sollten auch im Laufe der Therapie immer wieder angesprochen werden.

Frauen im gebärfähigen Alter sollten bestimmte Medikamente wie Valproat nicht erhalten.

- Die Planung von Schwangerschaft und Entbindung in Kooperation mit der Gynäkologie und die Nachbetreuung nach der Geburt gehören zu den wichtigen Aufgaben der Psychiaterin bzw. des Psychiaters.

\title{
Psychosen in Schwangerschaft und Stillzeit ${ }^{*}$
}

So chwangerschaft und Mutterschaft von Frauen, die an einer Psychose leiden oder gelitten haben, stellen Ärzte und Therapeuten zunehmend vor ganz neue Anforderungen, nicht zuletzt da über die Hälfte aller an einer Psychose erkrankten Frauen inzwischen Mütter werden $^{1,2}$. So sollten diese Patientinnen - wie alle psychisch kranken Frauen im geburtsfähigen Alter - immer wieder bezüglich Schwangerschaftswunsch angesprochen werden und eine spezielle Kontrazeptionsberatung erhalten. Bestimmte Psychopharmaka dürfen nicht oder nur mit äußerster Zurückhaltung verschrieben werden. Rechtzeitige Beratung und Planung in Bezug auf eine eventuelle Mutterschaft umfasst auch Beratung hinsichtlich einer möglichen Infertilität. Und schließlich geht es um die Behandlung von Psychosen in der Schwangerschaft und Postpartalzeit unter Berücksichtigung des Kindes, des Vaters und des weiteren Umfeldes.

Im Folgenden sollen verschiedene Problembereiche und Beratungssituationen im Einzelnen besprochen werden.

\section{Infertilität und Wunsch nach Mutterschaft}

Zunächst einmal können viele Frauen, die an einer chronischen oder rezidivierenden Psychose leiden und gerne Mutter werden wollen, sich diesen Wunsch nicht ohne Probleme erfüllen. Krank- heits- oder antipsychotikabedingt leiden sie an Infertilität. Manchmal fehlt auch ein geeigneter und zuverlässiger Partner. Ärzte und Ärztinnen sollten psychosekranke Frauen immer diesbezüglich befragen und beraten.

Besteht ein Kinderwunsch, so gilt es, die Gesamtsituation der Frau sorgfältig abzuwägen, möglichst unter Einbezug des Partners. Dabei sollte es zunächst einmal um die psychosoziale Situation der Frau gehen und die Partnerschaft, ihre Stabilität, die Wünsche des Partners etc. Hat sich das Paar über die zusätzliche Belastung durch ein Kind schon Gedanken gemacht und über die mögliche Unterstützung durch Bezugspersonen oder Betreuer, auch im Falle eines Rezidivs? Haben sie Fragen zur erwarteten Belastung des Kindes selbst, wie z. B. zu seinem genetischen Risiko?

Schließlich geht es auch um die Schwere und den voraussichtlichen Verlauf der Erkrankung. Ist die Patientin symptomfrei, und wie lange schon? Gab es in der Vorgeschichte viele Rezidive? Mutter und Vater sollten über das hohe Rückfallrisiko nach der Geburt aufgeklärt werden und darüber, wie diesem Risiko vorgebeugt werden kann.

Scheint eine Mutterschaft nach sorgältigem Abwägen möglich, so ist zu prüfen, ob der Krankheitsverlauf ein vorübergehendes Absetzen der antipsychotischen Medikation erlaubt. Dies wäre dann der Fall, wenn die Patientin mindestens ein

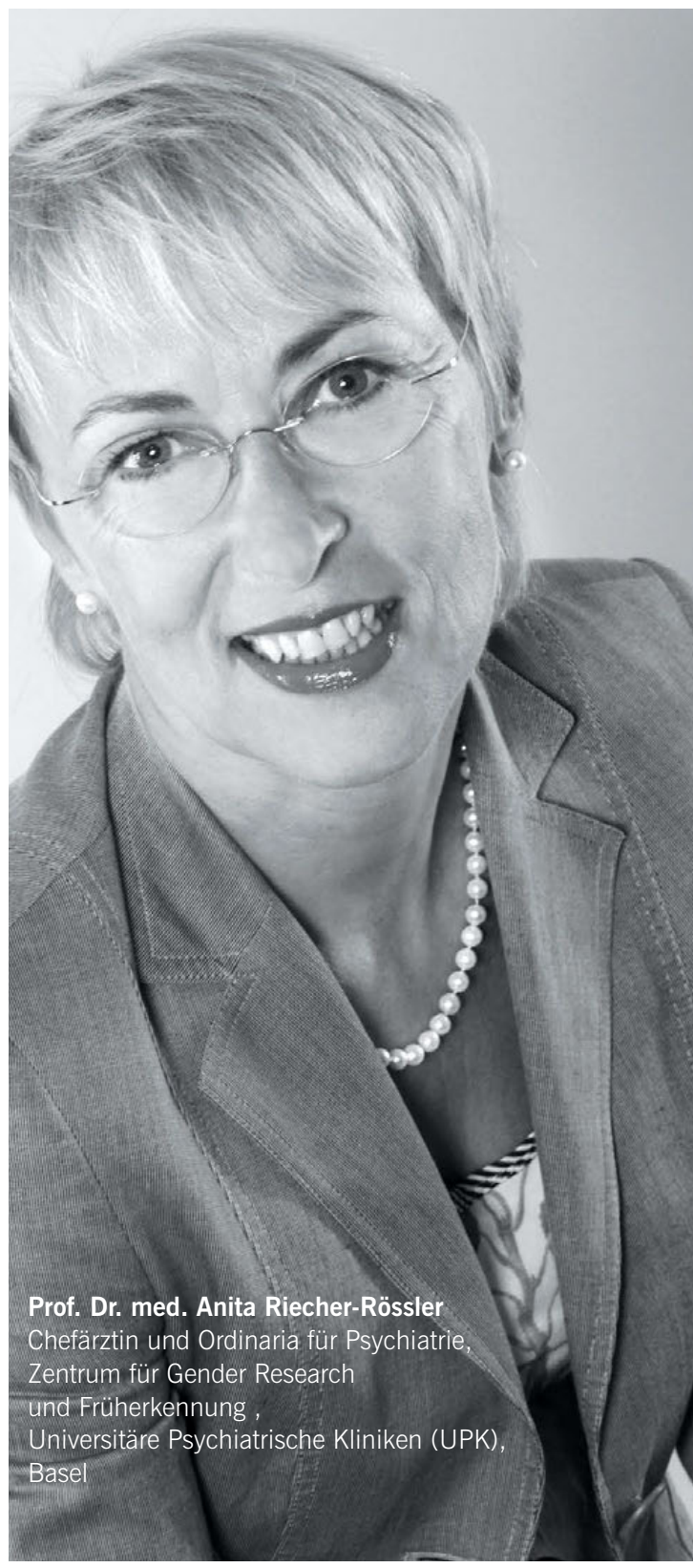

* Dieser Text wurde in Vorformen auch schon an anderer Stelle publiziert. 
bis zwei Jahre stabil und rückfallfrei war. In diesen Fällen kann die Medikation sehr langsam über Monate ausgeschlichen werden. Die Empfängnisverhütung sollte erst dann gestoppt werden, wenn die Patientin medikamentenfrei ist. Ist dies nicht möglich, so ist die Frau in Bezug auf die Einnahme von Psychopharmaka sorgfältig zu beraten. Diese sollten einerseits eine Empfängnis erlauben, andererseits in der Schwangerschaft sicher und gegebenenfalls auch kompatibel mit einem Stillwunsch sein (siehe unten).

Bei ungewollter Kinderlosigkeit müssen zunächst die Ursachen der Infertilität geklärt werden. Diese ist manchmal durch zu seltenen Geschlechtsverkehr, oft aber auch durch fehlenden Eisprung bedingt. Sowohl der mangelnden Libido als auch dem fehlenden Eisprung kann eine gonadale Dysfunktion zugrunde liegen. Stress kann - wie auch viele Antipsychotika zu einer Prolaktinerhöhung führen, und die Hyperprolaktinämie kann die Gonadenfunktion unterdrücken ${ }^{3}$.

\section{Ungeplante Schwangerschaft}

Laut einer australischen Studie waren über die Hälfte der Schwangerschaften bei psychosekranken Frauen ungewollt ${ }^{1}$. Die Gründe hierfür sind vielfältig. In neuerer Zeit führte oft die Umstellung von einem prolaktinerhöhenden auf ein sogenanntes prolaktinneutrales Antipsychotikum zu einer ungewollten Schwangerschaft, da durch die Hyperprolaktinämie der meisten älteren Antipsychotika kein Eisprung erfolgte und die Frau ohne Empfängnisverhütung auskam. Nach Umstellung auf ein prolaktinneutrales Antipsychotikum (Quetiapin, Clozapin, Aripiprazol oder auch Olanzapin) aber kehren sexuelle Lust und Fruchtbarkeit zurück, und die Frau wird schwanger, wenn sie bei Umstellung nicht bezüglich einer sicheren Empfängnisverhütung beraten wird. Dabei ist einem Intrauterinpessar der Vorzug zu geben, da hormonelle Kontrazeptiva zum Teil durch verstärkten Abbau bei gleichzeitiger Antipsychotika-Einnahme unsicher sind.

Die ungeplante Schwangerschaft wird oft

\section{"Das Risiko einer unbehandelten Psychose ist im Allgemeinen als deutlich höher einzuschätzen als das einer sorgfältig gewählten Psychopharmakotherapie."}

sehr spät entdeckt und der behandelnde Arzt mit der Frage konsultiert, ob die eingenommenen Medikamente zu kindlichen Fehlbildungen geführt haben könnten oder gar mit der Frage nach einem Schwangerschaftsabbruch. Frauen sind hier eingehend zu beraten (siehe unten).

\section{Psychose in der Schwangerschaft}

In der Schwangerschaft scheinen sich chronische Psychosen eher zu bessern, wahrscheinlich durch den in dieser Zeit erhöhten Östrogenspiegel. Trotzdem geht eine Psychose in der Schwangerschaft mit den verschiedensten Risiken einher. Im Extremfall wird die Schwangerschaft von der Kranken nicht erkannt oder verleugnet. Das Ungeborene kann durch komorbide Suchterkrankungen (Nikotin, Alkohol, Drogen) und unbedachte Medikamenteneinnahme geschädigt werden. Insbesondere scheinen diese Mütter häufig die Schwangerschaftsvorsorge nicht gut wahrzunehmen und insgesamt kein gutes Gesundheitsverhalten zu haben. Sie haben deshalb ein hohes Risiko für geburtshilfliche Komplikationen (Übersicht bei Barkla und McGrath4). Die wichtigsten Punkte der Behandlung von Psychosen in der Schwangerschaft fasst Tabelle 1 zusammen.

\section{Postpartale Psychosen}

Ein bis zwei von 1.000 Wöchnerinnen entwickeln in den ersten drei Monaten nach der Entbindung eine Psychose. Das größte Risiko birgt der erste Monat postpartal mit einem bis zu 20-fach (!) erhöhten Risiko im Vergleich zu vor der Schwangerschaft ${ }^{5}$.

Am häufigsten kommen nach der Entbindung offensichtlich depressive und manische Psychosen vor, aber auch solche mit einem schizoaffektiven oder schizophrenen Bild.

\section{Die Gefahren einer postpartalen Psycho- se sind:}

- Störung der Mutter-Kind-Beziehung und der Bindung

- Entwicklungsstörung des Kindes (emotional, kognitiv, Verhalten)

- Vernachlässigung/Misshandlung des Kindes

- Konflikte mit Partner/Betreuungspersonen

- Suizid, selten Infantizid (v. a. im Wahn)

\section{Tab. 1: Therapie der Psychose in der Schwangerschaft}

- Hospitalisierung (bei akuter psychotischer Symptomatik unumgänglich!)

- antipsychotische Medikation

- Stressreduktion

- Sicherstellung des Nachtschlafes

- Psychoedukation

- supportive Gespräche/Psychotherapie

- Beratung und praktische Hilfen

- soziale Maßnahmen

- Sicherstellung regelmäßiger geburtshilflicher Vorsorgeuntersuchungen!

- Planung der Entbindung und Postpartalzeit 
- Verzögerung des Hilfesuchens wegen mangelnder Krankheitseinsicht, Misstrauen, Furcht vor Stigmatisierung oder Furcht vor Verlust des Kindes

- Verzögerung der Diagnose und Behandlung wegen Nichterkennen der Symptome durch Geburtshelfer/ Hebammen

Die Therapie postpartaler Psychosen richtet sich weitgehend nach den allgemeinen Leitlinien der Behandlung der jeweiligen Psychose, hat aber die Besonderheiten der Postpartalsituation zu berücksichtigen (Tab. 2). Je nach klinischem Bild werden Antipsychotika, Antidepressiva und/oder Phasenprophylaktika eingesetzt.

Am besten sollte schon vor der Entbindung mit der Frau besprochen werden, ob sie stillen möchte. Zu bedenken ist hier zum einen, dass der Nachtschlaf vor allem in den ersten drei Monaten postpartal wegen des exzessiv hohen Rezidivrisikos unbedingt sichergestellt werden sollte. Zum anderen ist die Medikamentenauswahl bei stillenden Müttern wegen der potenziellen Risiken für den Säugling eingeschränkt. Es hat deshalb eine genaue Aufklärung mit Abwägen der Vor- und Nachteile des Stillens zu erfolgen. Entscheidet die Frau sich gegen das Stillen, so sollten beim Abstillen vorwiegend physikalische Maßnahmen angewandt werden. Das zu diesem Zweck zum Teil gegebene Bromocriptin ist ungeeignet, da es als Dopamin-Agonist die Psychose verstärken kann. Auch Antipsychotika, die eine Erhöhung des Prolaktins (siehe oben) und damit des Milchflusses bewirken, sollten möglichst vermieden werden.

Die medikamentöse Behandlung sollte stets von stützenden psychotherapeutischen Gesprächen, Beratung und praktischen Hilfen begleitet sein. Wichtig sind auch die Anleitung zum Umgang mit dem Kind, einschließlich der Babypflege, sowie eine engmaschige Supervision der Mutter-Kind-Interaktion.

Meist ist bei Psychosen eine stationäre Aufnahme erforderlich, bei fehlender

\section{Tab. 2: Therapie der postpartalen Psychose}

Hospitalisierung

\begin{tabular}{l|l} 
& $\begin{array}{l}\text { oder Fremdgefährdung (einschließlich Gefahr für } \\
\text { das Kind) } \\
\text { - später mit dem Baby in dafür spezialisierten } \\
\text { Einheiten }\end{array}$ \\
\hline $\begin{array}{l}\text { Medikation entsprechend } \\
\text { klinischem Bild }\end{array}$ & $\begin{array}{l}\text { - Antipsychotika } \\
\text { - Antidepressiva } \\
\text { - Phasenprophylaktika } \\
\text { - etc. }\end{array}$ \\
\hline $\begin{array}{l}\text { In schwersten, therapieresistenten } \\
\text { Fällen evtl. auch Elektrokrampf- } \\
\text { therapie }\end{array}$ & \\
\hline
\end{tabular}

\section{Stressreduktion}

Sicherstellung des Nachtschlafes

Psychoedukation

supportive Psychotherapie

Beratung und praktische Hilfen

Entlastung von anstrengenden Aufgaben wie der Dauerversorgung des Kindes

Supervision der Mutter-Kind-Interaktion und Einschätzung der elterlichen Fähigkeiten (,parenting assessment“)

Anleitung zum Umgang mit dem Kind (,parenting training“) und Förderung der Mutter-Kind-Beziehung

Planung der Nachbetreuung
- zunächst allein bei akuten Psychosen, bei Selbstoder Fremdgefährdung (einschließlich Gefahr für

später mit dem Baby in dafür spezialisierten

- Phasenprophylaktika

- etc
- Einbeziehung des Partners und der Angehörigen

- Einbeziehung des Partners und der Angehörigen

- Einbeziehung und Vernetzung professioneller Helfer: Hebamme, häusliche Pflege, Familienbetreuung, Sozialarbeiter, Gynäkologe, Hausarzt, Kinderarzt, falls notwendig Kindesschutz etc.
Tab. 3: Beurteilung und Training der Elternfähigkeiten - „parenting assessment“ und „parenting training“

\section{Beurteilung}

- Gibt es eine Gefahr für das Baby?

- Wie sorgt die Mutter für das Baby?

- Kann sie die Bedürfnisse des Babys erkennen und adäquat reagieren?

- Wie ist die emotionale Bindung? Ist sie eine „hinreichend gute Mutter“?

- Welche Hilfen braucht die Mutter? Wer muss involviert werden?

- Wie sind die Elternfunktionen des Vaters und gegebenenfalls anderer signifikanter Betreuungspersonen?

\section{Training und Förderung der Mutter-/Vater-Kind-Beziehung}

- Training der Versorgung des Babys

- Training der Interaktion mit dem Baby

- Training von „Beruhigen“, „Grenzen setzen“ etc

- Bindung stärken

- Eigene Krankheitsbewältigung/Behandlungscompliance bei gleichzeitiger Mutterschaft fördern

- Training des Vaters und gegebenenfalls anderer signifikanter Betreuungspersonen 


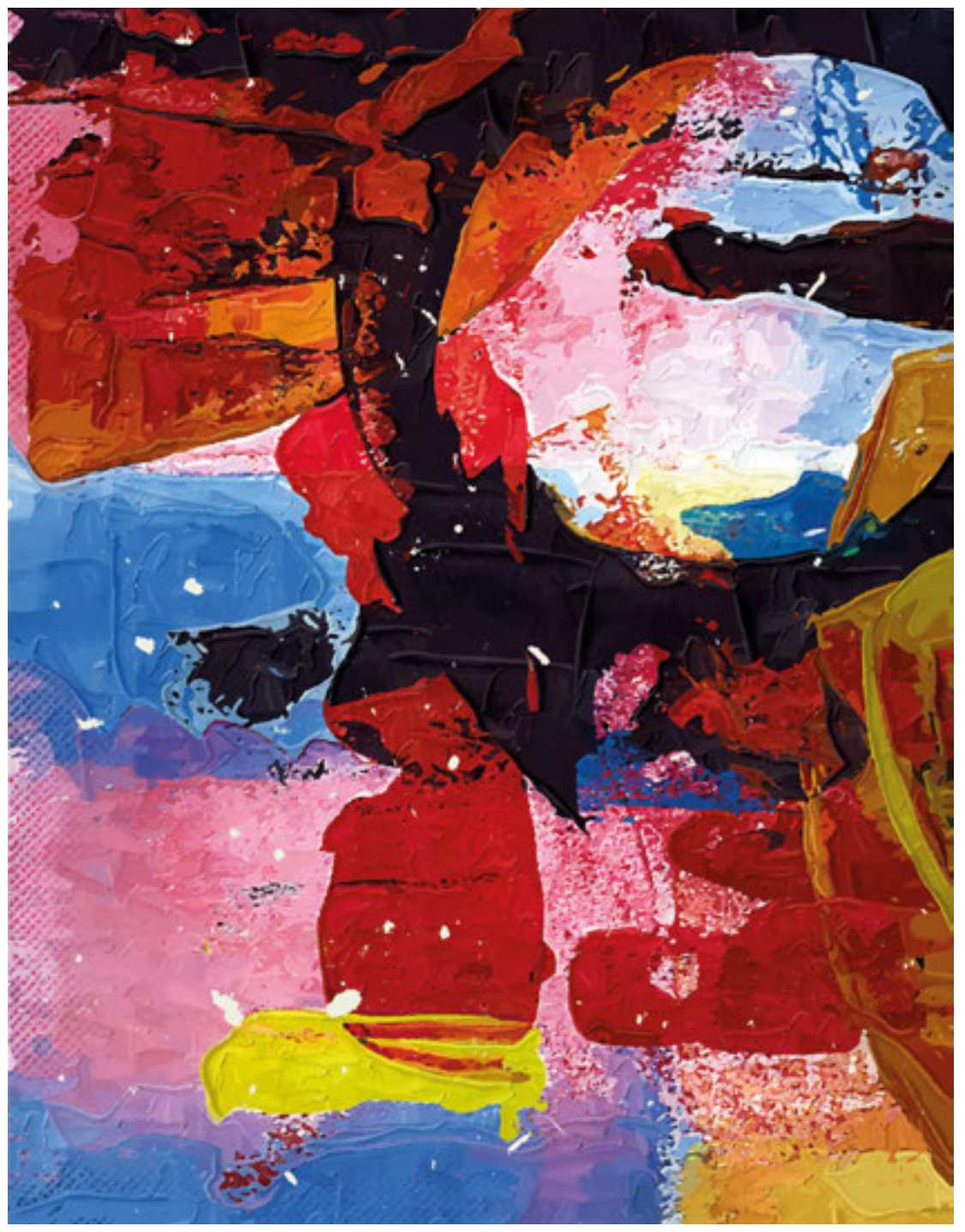

Krankheitseinsicht im Notfall auch über eine Zwangseinweisung, insbesondere wenn das Risiko einer Kindstötung besteht. Letztere ist zwar selten, aber etwa im Rahmen eines Wahns durchaus möglich. Die Hospitalisation sollte möglichst auf einer Mutter-Kind-Abteilung erfolgen, wo die Möglichkeit besteht, das Kind mit aufzunehmen, sobald der Zustand der Mutter dies erlaubt. Hier kann ein gutes „parenting assessment“ erfolgen, verbunden mit einem "parenting training“ (Tab. 3)

\section{Psychopharmakologie}

Bezüglich der Psychopharmakologie in Schwangerschaft und Stillzeit sei auf die einschlägige Literatur verwiesen ${ }^{6-9}$. Hier werden nur einige allgemeine Prinzipien besprochen.
Bestimmte Psychopharmaka mit erwiesenem Risiko der Teratogenität, insbesondere Valproat und die meisten anderen Phasenprophylaktika, sind bei Frauen im reproduktionsfähigen Alter ganz zu vermeiden. Äußert eine Frau, die potenziell teratogene Medikamente einnimmt, einen Schwangerschaftswunsch, ist eine medikamentenfreie Zeit für die Konzeption und bis zur Beendigung des dritten Trimenons anzustreben. Alternativ kann auf andere Psychopharmaka umgestellt werden.

Wird eine Frau während der antipsychotischen Behandlung schwanger, so gilt es, die Medikation nicht überstürzt abzusetzen, falls die Patientin gut eingestellt ist. Generell ist immer das erhöhte Rückfallrisiko bei der Umstellung gegen das potenzielle Risiko bei Beibehaltung eines bewährten Medikamentes abzuwägen.
Hat die werdende Mutter ein potenziell teratogenes Medikament in den ersten 12 Wochen post menstruationem genommen, empfiehlt sich eine UltraschallFeindiagnostik zum Ausschluss möglicher Fehlbildungen.

Das Risiko einer unbehandelten Psychose ist im Allgemeinen als deutlich höher einzuschätzen als das einer sorgfältig gewählten Psychopharmakotherapie. In der Schwangerschaft ist bei der Wahl eines Psychopharmakons nicht nur die mögliche Teratogenität mancher Psychopharmaka im ersten Trimenon zu bedenken, sondern auch die späteren perinatalen Komplikationen, wie unerwünschte Medikamenteneffekte, postnatale Entzugssymptome und Anpassungsprobleme beim Ungeborenen sowie schließlich potenzielle Langzeitkonsequenzen.

Während des Stillens ist die Pharmakaexposition generell deutlich niedriger als während der Schwangerschaft, auch wenn letztlich alle Psychopharmaka mehr oder weniger in die Muttermilch übertreten und die Verstoffwechselung beim Neugeborenen zum Teil noch langsam erfolgt, was wiederum die Medikamentenspiegel erhöhen kann.

Weitere Empfehlungen sind Tabelle $4 \mathrm{zu}$ entnehmen.

\section{Spezifische Probleme und Bedürfnisse von Müttern mit Psychosen}

Für eine psychosekranke Frau kann die Mutterschaft enormen Stress bedeuten, nicht nur wegen der Versorgung des Kindes. Viele Mütter haben auch keine feste Partnerschaft oder der Partner ist selbst nicht stabil genug, Unterstützung zu geben. Manchmal gibt es häusliche Gewalt, der Rechnung zu tragen ist ${ }^{10}$. Nicht selten kommen in der Herkunftsfamilie psychische Erkrankungen vor. All diese Stressoren können bei der Mutter die Erkrankung verstärken. Trotzdem suchen Mütter häufig keine Hilfe aufgrund der großen Angst, das Kind werde innen dann weggenommen. Es kommt zu einer Art Teufelskreis. Hier ist dringend niederschwellige professionelle Hilfe und $>$ 


\section{Tab. 4: Psychopharmaka-Gabe in der Schwangerschaft und Perinatalzeit}

Wichtigste Risiken
- Teratogenität (erste 12 Kalenderwochen post menstruationem)

- perinatale Komplikationen (unerwünschte Wirkungen der Medikamente, Entzugssymptome, andere Anpassungsstörungen)

- Verhaltensteratogenität und Langzeitfolgen?

Abwägen der Vor- und Nachteile!

Psychotherapie und alle anderen nichtmedikamentösen Behandlungsmaßnahmen ausschöpfen!

Vorsichtsmaßnahmen bei notwendiger Medikation: Ungeborenem/Neugeborenem
- gute Überwachung von Mutter und

- bei Einnahme im ersten Trimenon: Ultraschall-Feindiagnostik

- wenn möglich Monotherapie

- gegebenenfalls Dosisanpassung im zweiten und dritten Trimenon bei Medikamenten mit Clearance-Steigerung (z. B. Lithium, Lamotrigin)

- gegebenenfalls langsame Reduktion der Medikamente vor der Geburt, um Entzugssymptome bei Neugeborenen zu minimieren; unmittelbar nach der Geburt Dosissteigerung

- sorgältige interdisziplinäre Planung von Entbindung und Wochenbettbetreuung

- Beobachtung des Neugeborenen auf Anpassungsstörungen

modifiziert nach Riecher-Rössler $2012^{3}$

psychiatrisch-psychotherapeutische Begleitung nötig2, 3, 11 .

Ein umfassendes Betreuungsnetz ist zu etablieren, bestehend aus Familienmitgliedern, Freunden, Nachbarn und professionellen Helfern, wobei auf die Bedürfnisse dieser Betreuer durch spezielle Aufklärung, Training, laufende Supervision und Koordination eingegangen werden sollte. Wichtig ist dabei, dass es einen Hauptverantwortlichen im Sinne eines Case Managements gibt, der die Bedürfnisse von Mutter und Kind im Auge behält ${ }^{3}, 12$.

\section{Ätiologie und Pathogenese}

Was die Pathogenese der sogenannten peripartalen Psychosen betrifft, so handelt es sich prinzipiell um dieselben Erkrankungen, wie sie auch unabhängig von einer Entbindung auftreten können. Als stärkster Risikofaktor gilt eine vorbestehende Prädisposition, die sich etwa in Form einer familiären Belastung oder in einer eigenen früheren Erkrankung gezeigt haben kann. Die exzessive Risikosteigerung nach der Entbindung dürfte unter anderem mit dem starken Abfall des Ös-
trogen-Serumspiegels in dieser Zeit zusammenhängen. So ist der Östrogenspiegel während der Schwangerschaft auf etwa das 100-Fache der Norm erhöht, um dann nach der Entbindung innerhalb weniger Tage wieder auf den Normalwert zu fallen. Bei stillenden Frauen bleiben die Östrogenwerte niedrig. Östrogene modulieren aber die verschiedensten Hirnfunktionen und haben wahrscheinlich einen antipsychotischen Effekt ${ }^{13}$.

\section{Prophylaxe für Mutter und Kind}

Über das Risiko einer peripartalen Psychose sollten Schwangere - ebenso wie über das Risiko eines Blues oder einer postpartalen Depression ${ }^{14}$ - prophylaktisch aufgeklärt werden, am besten durch ihre Ärzte und in Geburtsvorbereitungskursen. Dies gilt insbesondere, wenn bei der Patientin oder in ihrer Familie schon einmal eine Psychose aufgetreten ist. Durch eine engmaschige Begleitung und Beobachtung in der Schwangerschaft und nach der Entbindung sowie eine frühzeitige Behandlung lässt sich die Rückfallhäufigkeit deutlich senken. Unbehandelt liegt das Rückfallrisiko bei Frauen, die schon einmal an einer Psychose gelitten haben, nach der Geburt etwa bei 25-50\%15, 16. Bei innen sollte unbedingt unmittelbar nach der Geburt mit einer Antipsychotika-Prophylaxe begonnen werden. Lehnen sie die stationäre Aufnahme ab, sollte unbedingt eine engmaschige Nachsorge mit Hausbesuchen und Supervision der mütterlichen Funktionen erfolgen.
1 Hearle J, McGrath J, Motherhood and schizophrenia. In: Castle D, McGrath J, Kulkarni J (Hrsg.): Women and schizophrenia. Cambridge, UK, Cambridge University Press, 2000; 79-94

2 Seeman M, Schizophrenia and motherhood. In: Göpfert M, Webster J, Seeman M (Hrsg.): Parental psychiatric disorder. Cambridge, Cambridge University Press, 2004

3 Riecher-Rössler A, Psychose in Schwangerschaft und Stillzeit. In: Riecher-Rössler A (Hrsg.): Psychische Erkrankungen in Schwangerschaft Riecher-Rossler A (Hrsg.): Psychische 2rkrankung

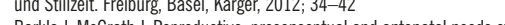
Barkla J, McGrath J, Reproductive, preconceptual and antenatal needs women with schizophrenia. In: Castle D, McGrath J, Kulkarni J (Hrsg.) Women and schizophrenia. Cambridge, UK, Cambridge University Press, 2000; 67-78

Kendell RE, Chalmers JC, Platz C, Epidemiology of puerperal psychoses. Brit J Psychiatry 1987; 150:662-673
6 Arzneimittelsicherheit in Schwangerschaft und Stillzeit. Accessed at: www.embryotox.de

Rohde A, Schaefer C, Psychopharmakotherapie in Schwangerschaft und Stillzeit. Arzneisicherheit - Beratung - Entscheidungsfindung. 3. vollständig überarb. u. erw. Aufl., Stuttgart, Thieme, 2010

8 Riecher-Rössler A, Heck A, Psychopharmakotherapie in Schwangerschaft und Stillzeit. In: Riecher-Rössler A (Hrsg.): Psychische Erkrankungen in Schwangerschaft und Stillzeit. Freiburg, Basel, Karger, 2012;69-89

9 Sutter A-L, Riecher-Rössler A, Psychotropic drugs and the perinatal sutter A-L, Riecher-Rossler A, Psychotropic drugs and the perinatal Perinatal Psychiatry. Heidelberg Springer, in print

10 Nyberg E, Riecher-Rössler A, Psychische Belastung durch häusliche

Nyberg E, Riecher-Rössler A, Psychische Belastung durch h
Gewalt. In: Boothe B, Riecher-Rössler A (Hrsg.): Frauen in Gewalt. In: Boothe B, Riecher-Rössler A (Hrsg.): Frau
Psychotherapie. Stuttgart, Schattauer, 2013; 83-92

11 Wimmer-Puchinger B, Psychische Störungen im Zusammenhang mit
Schwangerschaft und Geburt. In: Boothe B, Riecher-Rössler A (Hrsg.): Frauen in Psychotherapie: Grundlagen - Störungsbilder - Behandlungsangebote. Stuttgart, Schattauer, 2013; 239-252

12 Riecher-Rössler A, Gender-Aspekte. In: Rössler W, Kawohl W (Hrsg.): Soziale Psychiatrie: das Handbuch für die psychosoziale Praxis. Stuttgart, Kohlhammer, 2013; 127-141

13 Riecher-Rössler A, Kuhl H, Bitzer J, Psychische Störungen in Zeiten hormoneller Umstellungen - eine selektive Übersicht. Neuropsychiatr hormoneller Umstell

2006,20.155-165 Riecher-Rossler A, Depression in der Peripartalzet

und Prophylaxe. PSYCH up2date 2015; 9:149-160 Riecher-Rössler A, Psychische Störungen und Erkrankun
Entbindung. Fortschr Neurol Psychiatr 1997; 65:97-107

16 Fallgatter AJ et al., Klinische Aspekte der Wochenbettpsychosen

Übersicht mit drei Fallbeispielen. Nervenarzt 2002; 73:680-685 